\title{
Molecular mechanisms for biological endpoints of n-3 fatty acids
}

\author{
Richard J. Deckelbaum ${ }^{1,2}$, Chuchun Chang', Tilla S. Worgall ${ }^{1-3}$ \\ and Toru Seo ${ }^{1,2}$
}

'Institute of Human Nutrition, and Departments of ${ }^{2}$ Pediatrics and ${ }^{3}$ Pathology, Columbia University Medical Center, New York, USA

Abstract

Accumulating evidence in both humans and animal models clearly indicates that a group of very long-chain polyunsaturated fatty acids, the n-3 (or omega-3) fatty acids, have distinct and important bioactive properties compared with other groups of fatty acids. The n-3 fatty acids are known to reduce many risk factors associated with several diseases, such as cardiovascular diseases, diabetes and cancer. The mechanisms whereby n-3 fatty acids affect gene expression are complex. As examples, n-3 fatty acids regulate two groups of transcription factors, sterol regulatory element binding proteins and peroxisome proliferator activated receptors, which are critical for modulating the expression of genes controlling both systemic and tissuespecific lipid homeostasis. Modulation of specific genes by n-3 fatty acids and cross-talk between these genes are responsible for many effects of n-3 fatty acids.

Keywords: atherosclerosis; gene expression; $n-3$ fatty acids; SREBP

Abbreviations: ACAT: acyl cholesterol acyl transferase; DHA: docosahexaenic acid; EPA: eicosapentaenoic acid; LDL: low-density lipoprotein; nSREPB: nuclear SREBP; PPAR: peroxisome proliferator activated receptor; pSREBP: precursor SREBP; PUFA: polyunsaturated fatty acid; SRE: sterol regulatory element; $S R E B P$ : sterol regulatory element binding protein.

\section{Introduction}

$\mathrm{O}$ mega-3 (n-3) fatty acids are increasingly recognized to provide benefits in terms of health, disease prevention and disease treatment. As reviewed elsewhere (1) and in other papers included in this symposium, "Novel aspects of fatty acids: nutrition and biological function", n-3 fatty acids have key roles throughout the human life cycle. Beginning with pregnancy, where they have positive effects in increasing the gestational period, through infancy and, probably, later childhood, n-3 fatty acids have important roles in visual development and cognitive development and learning. Throughout the life cycle, n-3 fatty acids act as immune/inflammatory regulators with an overall link to diminishing adverse inflammatory effects and promoting positive immune endpoints. During adulthood and older age, reduction in risks associated with some cancers, metabolic syndrome and cardiovascular disease are described. Their roles in decreasing effects of mental disorders, and neurological and visual deterioration associated with aging are being reported. Because of the inefficient conversion of the essential n-3 fatty acid precursor, 18:3 ?-linolenic acid, to the more potent bioactive forms of n-3 fatty acids, eicosapentaenoic acid (EPA) and docosahexaenoic acid (DHA), most studies are reporting that beneficial effects require increasing intakes of EPA and DHA directly, rather than from the n-3 18:3 precursor (1).

\section{Biological actions}

The $\mathrm{n}-3$ fatty acids are very potent modulators of a large number of cell biology and physiological pathways $(1,2)$. These fatty acids have distinct effects on cell membrane structure and function compared with other fatty acids. In general, n-3 fatty acids lead to the production of anti-inflammatory cytokines and eicosanoids, whereas n- 6 fatty acids are more linked to proinflammatory pathways. The n-3 fatty acids affect signaling pathways, cell proliferation, cell differentiation and expression of a number of receptors, and regulate the activity of a number of enzymes. They also improve vascular 
resistance in animals and humans, and decrease the level of blood coagulation pathways by influencing both clotting factors and platelet activity (1).

The $\mathrm{n}-3$ fatty acids are important modulators of a number of different gene families related to inflammation, lipid metabolism and energy utilization (2). Examples of inflammatory protein and genes related to suppression by n-3 fatty acids include cylooxygenases, interleukins, tumor necrosis factor- $\alpha$ and many others. In terms of lipid metabolism and energy utilization, n-3 fatty acids are potent regulators of foxhead proteins, peroxisome proliferator activated receptors (PPARs) and sterol regulatory element binding proteins (SREBPs), among others. Like other nutrients, n-3 fatty acids affect the expression or processing of intermediates in gene expression such as transcription factors, nuclear hormone receptors and second messengers, which then serve to trigger cascades important in a number of disease conditions and cell biological endpoints, as summarized above (2).

\section{From molecular mechanisms to application}

A major area of interest in recent years has been the ability of n-3 fatty acids to affect blood lipid levels and dyslipidemia. More recently, they have been shown to play a distinct role in cardiovascular disease, insulin resistance and hepatic steatosis. In addition, it was recently found that n-3 fatty acids are potent inhibitors of arterial cholesterol delivery, an early pathway in the progression of atherosclerosis. Cholesterol and polyunsaturated fatty acids (PUFAs) are frequent partners in regulating lipid physiology. For example, both high levels of dietary cholesterol and saturated fatty acids suppress lowdensity lipoprotein (LDL) receptor activity and are associated with higher plasma levels of cholesterol. Cholesterol and fatty acids partner in sterol esterification to convert free cholesterol to cholesteryl ester via the enzyme acyl cholesterol acyl transferase (ACAT). Oleic acid and linoleic acid lower LDLcholesterol levels and one contributing mechanism to this may be their ability to esterify free cholesterol, via the ACAT reaction, leading to a need to up-regulate the LDL receptor because of decreasing availability of intracellular free cholesterol (3). EPA and DHA are poor substrates for the ACAT reaction (4), and this raises one mechanistic possibility in explaining the relatively weak effect of these n-3 fatty acids on plasma LDL levels compared with shorter n-6 PUFAs.
The effects of n-3 fatty acids on SREBP gene expression are an example of how these fatty acids can affect multiple areas of lipid and energy metabolism. Three SREBPs are described: SREBP-1a and SREBP-1c, which largely regulate genes of fatty acid metabolism, and SREBP-2, which regulates many genes involved cholesterol metabolism (5). Of note, there is cross-over between the ability of both cholesterol and PUFAs to inhibit the processing of the inactive precursor form of SREBP, pSREBP, to its mature active nuclear form, nSREBP (6). Longer chain PUFAs, as exemplified by DHA, are especially powerful in decreasing sterol regulatory element (SRE)-mediated gene expression. Several groups have defined mechanisms whereby n-3 fatty acids lead to decreased levels of pSREBP and its effects on gene expression (6-9). These include processes leading to the decreased levels of both the mature the precursor form of SREBP, pSREBP, and diminished conversion of the inactive precursor form via proteolytic cleavage to the active form (6). Studies in model membrane systems showed that fatty acids decrease the affinity of cholesterol for phospholipid, which leads to enhanced transfer from cholesterol-rich regions (e.g. the plasma membrane) to cholesterol-poor regions (e.g. the endoplasmic reticulum) (10); increased cholesterol in the endoplasmic reticulum would lead to decreased SREBP transport to the Golgi.

The authors' laboratory has also shown that PUFAs lead to enhanced sphingomyelinase activity, with resulting hydrolysis of plasma membrane sphingomyelin to ceramide (11). Since sphingomyelin has higher affinity for free cholesterol than other phospholipids, this effect is likely to result in more free cholesterol being available in the endoplasmic reticulum, again inhibiting SREBP processing. Separately, it was found that ceramide itself markedly inhibits SPREBP processing via effects on sphingolipid synthesis and inhibiting vesicular trafficking between the endoplasmic reticulum and the Golgi (12). Thus, n-3 fatty acids can act at a number of points that will affect and decrease SREBP expression and processing.

The ability of $n-3$ fatty acids to activate PPARs and associated stimulants of fatty acid oxidation, along with their ability to decrease SREBP-1c processing and expression, probably contributes to their ability to lower plasma triglyceride levels (13, 14). These combined effects, for example, help us to 
understand recently observed experiments where chronic feeding of n-3-rich diets to wild-type and insulin-resistant mice led to marked effects of n-3 fatty acids, lowering both normal (wild-type mice) and elevated (insulin-resistant mice) plasma triglyceride levels (C Chung, T Seo, RJ Deckelbaum, unpublished observations). In addition, in the insulin-resistant mice, there was a very marked diminished decrease in liver triglyceride accumulation, accompanied by lower expression of SREBP1c protein.

Experiments comparing the effect of chow feeding with diets high in saturated fat versus n-3 fatty acid are defining the effects important to the early development of atherosclerosis. A Western-type diet rich in saturated fat increases LDL-cholesterol deposition in the arterial wall substantially and this is linked to increases in aortic expression and activity of lipoprotein lipase (15). Lipoprotein lipase, in addition to its catalytic activity on triglycerides, is an important bridging or anchoring molecule for LDL to cell surfaces, and this results in increased delivery of LDL-cholesterol into cells (16). In strong contrast, n-3 fatty acid feeding decreases LDL-cholesterol delivery to the arterial wall both in wild-type mice and insulin-resistant mice, and this is associated with decreased lipoprotein lipase expression in the arterial wall (C Chung, T Seo, RJ Deckelbaum, unpublished observations). Since these effects occur before the appearance of even fatty streaks in the arterial wall, n-3 fatty acids could be important in inhibiting an early step in atherosclerosis progression, i.e. cholesterol deposition in the arterial wall.

\section{Conclusions}

Biologically favorable effects of EPA and DHA are probably mediated through effects on several distinct pathways within cells, tissues and organs. These include direct interactions in changing the composition of cell membranes and membrane function, activating or suppressing signaling molecules, interacting directly with DNA as well as with proteins that affect the processing of transcription factors, and affecting enzyme activities and vesicular endoplasmic reticulum-Golgi trafficking. Overall, n-3 fatty acids are now recognized as important mediators of inflammatory/immune control pathways and of key steps in energy and lipid metabolism (Fig. 1). Expression of inflammatory proteins is usually suppressed, while energy-lipid metabolic

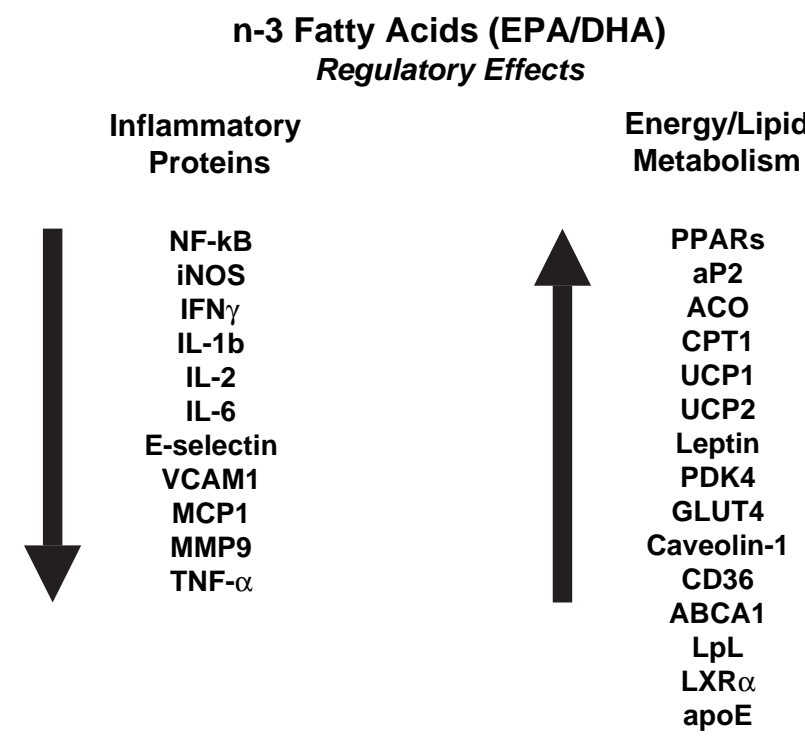

Fig. 1. Genes regulated by n-3 fatty acids. Representative genes affecting inflammatory processes and controlling energy and lipid metabolism are shown. Expression of proinflammatory genes is generally decreased (left). In contrast, genes regulating energy and lipid metabolism are generally activated (right). EPA: eicosapentaenoic acid; DHA: docosahexaenic acid; NK: nuclear factor; IFN: interferon; IL: interleukin; VCAM: vascular cell adhesion molecule; MCP: ??; MMP: ??; TNF: tumor necrosis factor; PPAR: peroxisome proliferator activated receptor; aP2: ??; ACO: ??; CPT: ??; UCP: ??; PDK: ??; GLUT: ??; ABCA: ??; LpL: lipoprotein lipase; LXR: liver $\mathrm{X}$ receptor; apo: apolipoprotein.[Adapted from Deckelbaum et al., 2006 (2).]

pathways are up-regulated by n-3 fatty acids (2). It was also recently suggested that because of their high content of double bonds, rather than being pro-oxidant, EPA and DHA may act as scavengers of reactive oxygen species. Some of the effects of EPA and DHA may not be related directly to the fatty acid molecule, but rather to their metabolites, such as eicosanoids or other molecules. Although it appears that EPA and DHA show similar bioactivity in many instances, frequent differences are described in potency between these n-3 fatty acids in different cells and tissues. Mechanisms underlying the specific responses by cells to EPA and DHA need to be better understood in order to anticipate better selectivity of DHA compared with EPA, not only in modulating key biological pathways and gene expression, but also in preventing and treating specific diseases, and moving towards better health outcomes.

\section{Acknowledgements}

This study was supported by NIH grant HL 40404 (RJ D), American Heart Association Grant-in-Aid 0255656N 
(TSW) and NIH grant DK60497 (TS). None of the authors had any conflicts of interest.

\section{References}

1. Seo T, Blaner WS, Deckelbaum RJ. Omega-3 fatty acids: molecular approaches to optimal biological outcomes. Curr Opin Lipidol 2005; 16: 11-8.

2. Deckelbaum RJ, Worgall TS, Seo T. n-3 Fatty acids and gene expression. Am J Clin Nutr 2006; 83(Suppl): 1520 $5 \mathrm{~S}$.

3. Rumsey SC, Galeano NF, Lipschitz B, Deckelbaum RJ. Oleate and other long chain fatty acids stimulate low density lipoprotein receptor activity by enhancing acyl coenzyme A: cholesterol acyl transferase activity and altering intracellular regulatory cholesterol pools in cultured cells. J Biol Chem 1995; 270: 10008-16.

4. Seo T, Oelkers PM, Giattina MR, Worgall TS, Sturley SL, Deckelbaum RJ, et al. Differential modulation of ACAT1 and ACAT2 transcription and activity by long chain free fatty acids in cultured cells. Biochemistry 2001; 40: 4756-62.

5. Horton JD, Shah NA, Warrington JA, Anderson NN, Park SW, Brown MS, Goldstein JL. Combined analysis of oligonucleotide microarray data from transgenic and knockout mice identifies direct SREBP target genes. Proc Natl Acad Sci USA 2003; 100: 12027-32.

6. Worgall TS, Sturley SL, Seo T, Osborne TF, Deckelbaum RJ. Polyunsaturated fatty acids decrease expression of promoters with sterol regulatory elements by decreasing levels of mature sterol regulatory elementbinding protein. J Biol Chem 1998; 273: 25537-40.

7. Thewke DP, Panini SR, Sinensky M. Oleate potentiates oxysterol inhibition of transcription from sterol regulatory element-1-regulated promoters and maturation of sterol regulatory element-binding proteins. J Biol Chem 1998; 273: 21402-7.

8. Hannah VC, Ou J, Luong A, Goldstein JL, Brown MS. Unsaturated fatty acids down-regulate SREBP isoforms 1a and 1c by two mechanisms in HEK-293 cells. J Biol Chem 2001; 276: 4365-72.

9. Xu J, Cho H, O’Malley S, Park JH, Clarke SD. Dietary polyunsaturated fats regulate rat liver sterol regulatory element binding proteins- 1 and -2 in three distinct stages and by different mechanisms. J Nutr 2002; 132: 3333-9.

10. Johnson RA, Hamilton JA, Worgall TS, Deckelbaum RJ. Free fatty acids modulate intermembrane trafficking of cholesterol by increasing lipid mobilities: novel ${ }^{13} \mathrm{C}$ NMR analyses of free cholesterol positioning. Biochemistry 2003 ; 42: 1637-45.

11. Worgall TS, Johnson RA, Seo T, Gierens H, Deckelbaum RJ. Unsaturated fatty acid-mediated decreases in sterol regulatory element-mediated gene transcription are linked to cellular sphingolipid metabolism. J Biol Chem 2002; 277: 3878-85.

12. Worgall TS, Juliano RA, Seo T, Deckelbaum RJ. Ceramide synthesis correlates with the posttranscriptional regulation of the sterol-regulatory element-binding protein. Arterioscler Thromb Vasc Biol 2004; 24: 943-8.

13. Jump DB. Dietary polyunsaturated fatty acids and regulation of gene transcription. Curr Opin Lipidol 2002; 13: 155-64.

14. Clarke SD. The multi-dimensional regulation of gene expression by fatty acids: polyunsaturated fats as nutrient sensors. Curr Opin Lipidol 2004; 15: 13-8.

15. Seo T, Qi K, Chang C, Liu Y, Worgall TS, Ramakrishnan R, Deckelbaum RJ. Saturated fat-rich diet enhances selective uptake of LDL cholesteryl esters in the arterial wall. J Clin Invest 2005; 115: 2214-22.

16. Merkel M, Kako Y, Radner H, Cho IS, Ramasamy R, Brunzell JD, et al. Catalytically inactive lipoprotein lipase expression in muscle of transgenic mice increases very low density lipoprotein uptake: direct evidence that lipoprotein lipase bridging occurs in vivo. Proc Natl Acad Sci USA 1998; 95: 13841-6.

Richard J. Deckelbaum, MD

Institute of Human Nutrition

Columbia University Medical Center

630 West 168th Street

$\mathrm{PH} 15-1512$

New York

NY 10032, USA

Tel: + I 2123054808

Fax: + I 2123053079

E-mail: rjd20@columbia.edu 